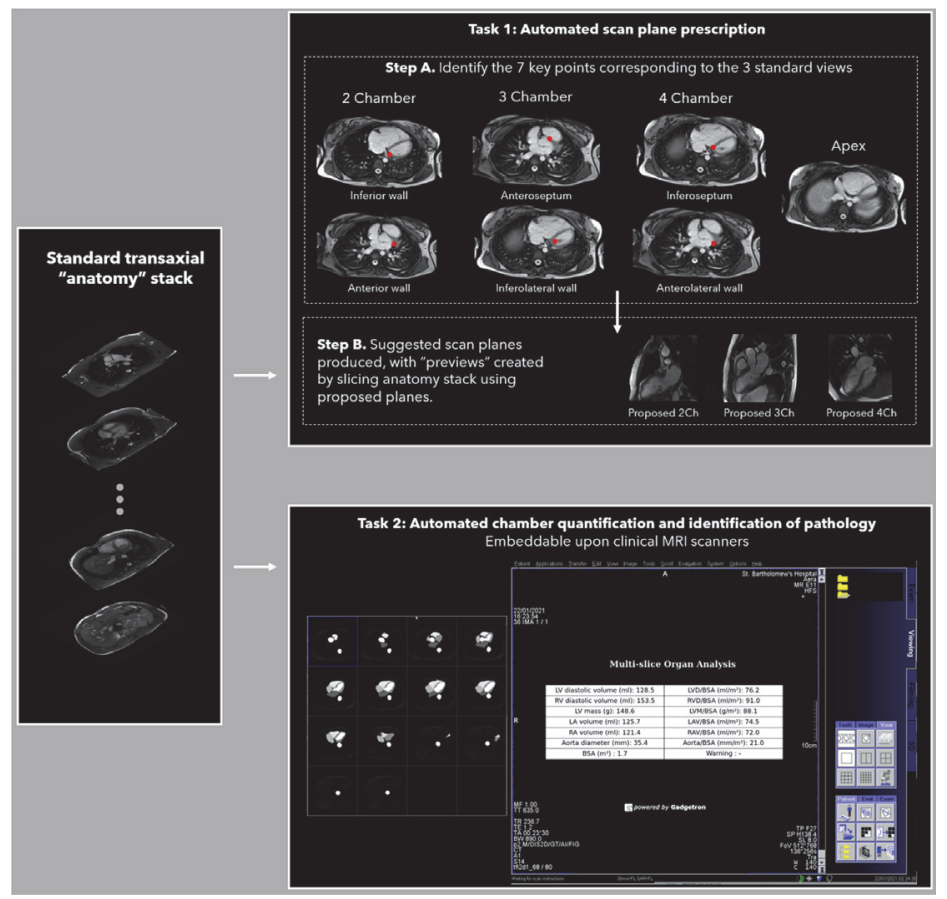

obtained from the full scans. 200 scans were used as a testing set.

Results Task 1: The network successfully learned the 7 key points on the transaxial stack, and the scan planes it proposed closely matched those chosen by radiographers using dedicated localisers, with a median out-of-plane distance at the key points of only $3.4 \mathrm{~mm}$ (IQR 1.6-6.3) - less than half the slice thickness $(8 \mathrm{~mm})$ of the gold standard cines.

Task 2: The system was successful in segmenting the transaxial slices (Dice 0.91). Diagnostic accuracy was high for abnormalities including left and right ventricular dilatation (90.5\%, 85.5\%), LVH (85\%) and ascending aorta dilatation (94.4\%). The ROC AUC for diagnosing pleural effusions was 0.91 .

Conclusion A neural network can use the standard transaxial stack of images to identify important pathology and accurately predict scan planes close to those of expert radiographers using dedicated localisers. This system could speed up the delivery of CMR and lead to faster and more dynamic scanning protocols.

\section{IDENTIFICATION OF THIRTY NOVEL LOCI FOR CARDIOVASCULAR MAGNETIC RESONANCE DERIVED AORTIC DISTENSIBILITY IN THE UK BIOBANK}

\footnotetext{
1,2Kenneth Fung, ${ }^{3}$ Luca Biasiolli, ${ }^{3}$ Evan Hann, ${ }^{3}$ Elena Lukaschuk, ${ }^{1}$ Julia Ramírez, ${ }^{1}$ Stefan van Duijvenboden, ${ }^{1,2}$ Nay Aung, 'Jose M Paiva, ${ }^{1}$ Mihir M Sanghvi, 'Ross J Thomson, ${ }^{1}$ Aaron M Lee, ${ }^{3}$ Stefan K Piechnik, ${ }^{3}$ Stefan Neubauer, ${ }^{1,2}$ Steffen E Petersen, ${ }^{1}$ Patricia B Munroe. 'William Harvey Research Institute, NIHR Barts Biomedical Research Centre, Queen Mary University of London, Charterhouse Square, London, UK; ${ }^{2}$ Barts Heart Centre, St Bartholomew's Hospital, Barts Health NHS Trust, London, UK; ${ }^{3}$ Division of Cardiovascular Medicine, Radcliffe Department of Medicine, National Institute for Health Research Oxford Biomedical Research Centre, Oxford University Hospitals National Health Service Foundation Trust, University of Oxford, Oxford, UK
}

10.1136/heartjnl-2021-BSCMR.9
Introduction Cardiovascular magnetic resonance (CMR)-derived aortic distensibility (AoD) is a validated tool to measure arterial stiffness ${ }^{1}$. This local stiffness marker is an independent predictor of cardiovascular events and all-cause mortality ${ }^{2}$. Our knowledge of genes modulating aortic stiffness remains limited. We conducted genome-wide association studies (GWASs) for AoD in the ascending aorta (AA) and proximal descending aorta (PDA).

Methods 34,039 UK Biobank (UKB) participants of European ancestry with transverse cine images of pulmonary trunk and right pulmonary artery acquired using a $1.5 \mathrm{~T}$ CMR scanner were included. AoD is defined as the relative change in crosssectional aorta area for a given change in central pulse pressure, they were either manually derived or processed through a developed automated algorithm ${ }^{3}$. GWASs were performed using linear mixed models ${ }^{4}$ using $\sim 7$ million imputed variants adjusted for age, sex, height, weight, systolic blood pressure, aortic area segmentation method (manual/automated), imaging centre, genotype array, smoking, diabetes mellitus and top 10 principal components. Bioinformatic analyses were performed including investigation of trait pleiotropy. Polygenic risk scores (PRS) for AA and PDA were calculated using PRSice ${ }^{5}$ and tested for association with major adverse cardiovascular events (MACE) in the UKB cohort without imaging data $(n=$ 98,559).

Results and discussion Variants at 18 independent loci for AA and 16 loci for PDA AoD were genome-wide significant, $\mathrm{P}<$ $5 \times 10^{-8}$ (table 1 and figure 1) with four shared loci between the two phenotypes. The heritability for both AA and PDA AoD was $\sim 27 \%$, much higher than arterial stiffness index $(6 \%)^{6}$ but comparable to pulse wave velocity (36$40 \%)^{7,8}$. We captured a modest percentage of the genetic variance for each trait $(2.7 \%$ for AA; $2.1 \%$ for PDA). Several candidate genes are highlighted including ELN for both traits, with ISL1 and PCSK1 both involved in insulin regulation that may explain the interplay with diabetes ${ }^{9,10}$. Variants at 13 loci were significant with blood pressure and/or coronary artery 


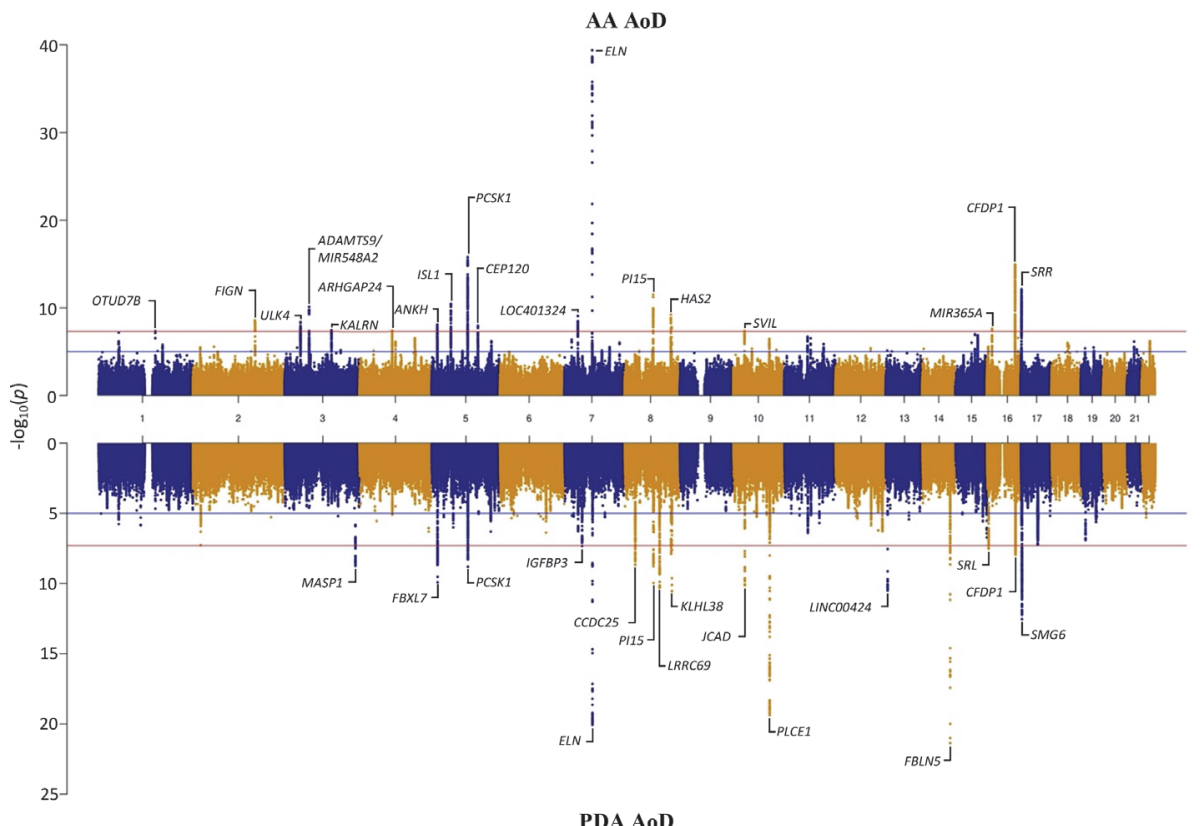

Abstract 9 Figure 1 Miami plots of genome-wide association test statistics for aortic distensibility in the ascending (AA AoD) and descending aorta $(\mathrm{PDA} A \mathrm{oD})$. The red line indicates the $P$-value threshold for genome-wide significance $\left(5 \times 10^{-8}\right)$ while the blue line indicates $P$-value threshold for suggestive significance $\left(1 \times 10^{-5}\right)$

Abstract 9 Table 1 Genome-wide significant loci for aortic distensibility in the ascending aorta $(\mathrm{n}=33,346)$ and proximal descending aorta (n $=33,800$ )

\begin{tabular}{|c|c|c|c|c|c|c|c|c|c|c|c|c|c|c|}
\hline \multicolumn{15}{|c|}{ Ascending aortic distensibility } \\
\hline Locus & rsID & CHR & \multicolumn{2}{|c|}{ Position (build 37) } & \multicolumn{2}{|l|}{ EA } & EAF & $P$ & \multicolumn{2}{|l|}{$\mathrm{N}$} & \multicolumn{2}{|l|}{$\beta$} & \multicolumn{2}{|l|}{ SE } \\
\hline OTUD7B & rs67807996 & 1 & \multicolumn{2}{|l|}{149995265} & \multicolumn{2}{|l|}{ G } & 0.604 & 4.7E-08 & \multicolumn{2}{|c|}{31109} & \multicolumn{2}{|l|}{-2.20} & \multicolumn{2}{|l|}{0.41} \\
\hline FIGN & rs35303331 & 2 & \multicolumn{2}{|l|}{164921770} & \multicolumn{2}{|l|}{$A$} & 0.767 & 2.4E-09 & \multicolumn{2}{|c|}{33201} & \multicolumn{2}{|l|}{2.79} & \multicolumn{2}{|l|}{0.46} \\
\hline ULK4 & rs751328021 & 3 & \multicolumn{2}{|l|}{41971728} & \multicolumn{2}{|c|}{ TTTCCTATTTAATAGGAAATCCTATTGTG } & 0.870 & 4.0E-09 & 3151 & & -3.41 & & 0.59 & \\
\hline ADAMTS9-AS2I & rs7638565 & 3 & 64723072 & & $A$ & & 0.407 & 7.4E-11 & 3311 & & 2.60 & & 0.39 & \\
\hline MIR548A2 & & & & & & & & & & & & & & \\
\hline KALRN & rs4608635 & 3 & 123961496 & & $C$ & & 0.342 & 3.6E-08 & 3201 & & -2.26 & & 0.42 & \\
\hline ARHGAP24 & rs17399707 & 4 & 86716478 & & $\mathrm{C}$ & & 0.795 & 4.1E-08 & 3240 & & 2.71 & & 0.49 & \\
\hline ANKH & rs9686327 & 5 & 15018537 & & G & & 0.819 & $8.2 \mathrm{E}-09$ & 3293 & & -2.84 & & 0.50 & \\
\hline ISL1 & rs540082300 & 5 & 51196504 & & $\mathrm{C}$ & & 0.603 & $3.5 \mathrm{E}-11$ & 3293 & & 2.66 & & 0.40 & \\
\hline PCSK1 & rs764443335 & 5 & 95606712 & & AATG & & 0.645 & $1.6 \mathrm{E}-16$ & 3282 & & -3.29 & & 0.41 & \\
\hline CEP120 & rs115968765 & 5 & 122621332 & & $\mathrm{C}$ & & 0.732 & $1.1 \mathrm{E}-08$ & 3272 & & 2.53 & & 0.44 & \\
\hline LOC401324 & rs342983 & 7 & 35464784 & & $\mathrm{~T}$ & & 0.272 & 8.4E-10 & 3133 & & 2.78 & & 0.45 & \\
\hline ELN & rs7795735 & 7 & 73429482 & & $\mathrm{~T}$ & & 0.545 & $4.1 \mathrm{E}-40$ & 3304 & & 5.30 & & 0.39 & \\
\hline PI15 & rs9721183 & 8 & 75781818 & & $\mathrm{C}$ & & 0.634 & $3.0 \mathrm{E}-12$ & 3158 & & -2.83 & & 0.41 & \\
\hline HAS2 & rs563877611 & 8 & 122649630 & & $C$ & & 0.737 & $5.9 \mathrm{E}-10$ & 3071 & & -2.78 & & 0.46 & \\
\hline SVIL & rs3006557 & 10 & 30090348 & & $\mathrm{G}$ & & 0.283 & $4.5 \mathrm{E}-08$ & 3314 & & -2.33 & & 0.43 & \\
\hline MIR365A & rs7203968 & 16 & 14444230 & & $\mathrm{G}$ & & 0.833 & $2.3 \mathrm{E}-08$ & 3248 & & 2.96 & & 0.52 & \\
\hline CFDP1 & rs4888421 & 16 & 75465339 & & A & & 0.403 & $1.1 \mathrm{E}-15$ & 3308 & & -3.12 & & 0.40 & \\
\hline SRR & rs4548913 & 17 & 2209888 & & G & & 0.361 & $7.5 \mathrm{E}-13$ & 3297 & & -2.87 & & 0.41 & \\
\hline Descending aor & distensibility & & & & & & & & & & & & & \\
\hline Locus & rsID & & CHR & Posi & on (build 37) & EA & & EAF & $\mathbf{P}$ & $\mathrm{N}$ & & $\beta$ & & SE \\
\hline MASP1 & $3: 18$ & 5537_AT_A & 3 & 1869 & 5537 & AT & & 0.924 & $1.8 \mathrm{E}-09$ & 33222 & & 2.77 & & 0.46 \\
\hline FBXL7 & rs52 & 4362 & 5 & 1547 & 344 & G & & 0.664 & $1.2 \mathrm{E}-10$ & 31606 & & 1.69 & & 0.26 \\
\hline PCSK1 & rs 10 & 122 & 5 & 9561 & 520 & $\mathrm{~T}$ & & 0.550 & 1.6E-09 & 33468 & & -1.44 & & 0.24 \\
\hline IGFBP3 & $\mathrm{rs} 15$ & & 7 & 4600 & 323 & $C$ & & 0.546 & 4.6E-08 & 33560 & & -1.30 & & 0.24 \\
\hline ELN & rs 69 & & 7 & 7342 & 373 & $A$ & & 0.540 & $8.8 \mathrm{E}-21$ & 33772 & & 2.26 & & 0.24 \\
\hline CCDC25 & rs 56 & 913 & 8 & 2761 & 585 & A & & 0.558 & $2.2 \mathrm{E}-09$ & 33729 & & -1.42 & & 0.24 \\
\hline PI15 & rs 97 & & 8 & 7578 & 818 & $C$ & & 0.634 & 1.1E-10 & 32019 & & -1.62 & & 0.25 \\
\hline LRRC69 & $\mathrm{rs} 10$ & 555 & 8 & 9219 & 899 & $\mathrm{~T}$ & & 0.312 & $5.0 \mathrm{E}-11$ & 33520 & & 1.71 & & 0.26 \\
\hline KLHL38 & rs70 & & 8 & 1246 & 8614 & $\mathrm{~T}$ & & 0.635 & 2.7E-11 & 33333 & & 1.67 & & 0.25 \\
\hline JCAD & rs 23 & & 10 & 3016 & 031 & $\mathrm{~T}$ & & 0.319 & 7.3E-11 & 33689 & & -1.65 & & 0.26 \\
\hline
\end{tabular}




\begin{tabular}{|c|c|c|c|c|c|c|c|c|c|}
\hline PLCE1 & rs61886305 & 10 & 95902053 & c & 0.825 & $4.2 \mathrm{E}-20$ & 33447 & 2.94 & 0.32 \\
\hline LINC00424 & rs12869493 & 13 & 22872349 & c & 0.784 & $3.1 \mathrm{E}-11$ & 33413 & -1.91 & 0.29 \\
\hline FBLN5 & rs8014161 & 14 & 92393198 & $T$ & 0.639 & $4.3 \mathrm{E}-22$ & 33088 & -2.38 & 0.25 \\
\hline SRL & rs11864324 & 16 & 4281391 & $T$ & 0.779 & $3.0 \mathrm{E}-08$ & 33800 & -1.58 & 0.29 \\
\hline CFDP1 & rs3851734 & 16 & 75371920 & $\mathrm{~T}$ & 0.406 & $1.1 \mathrm{E}-08$ & 33636 & -1.38 & 0.24 \\
\hline SMG6 & rs1532292 & 17 & 2097483 & $T$ & 0.618 & $2.9 \mathrm{E}-13$ & 33768 & 1.81 & 0.25 \\
\hline
\end{tabular}

Locus indicates the name of the gene in closest proximity to the lead variant.

rsID = reference single nucleotide polymorphism cluster ID; CHR = chromosome; $E A=$ effect allele; $E A F=$ effect allele frequency; $P=P$-value $($ standard infinitesimal mixed model); $N=$ effective number of participants; $\beta=$ effect-size estimate expressed as a percentage; $\mathrm{SE}=$ standard error.

disease traits. We observed no significant difference in odds ratio for MACE between the top and bottom quintiles for each PRS.

Conclusion We identified 30 genetic loci providing new candidate genes for exploration of biological mechanism of AoDs.

\section{REFERENCES}

1. Nelson A, Worthley S, Cameron J, Willoughby S, Piantadosi C, Carbone A, Dundon B, Leung M, Hope S, Meredith I, Worthley M. Cardiovascular magnetic resonance-derived aortic distensibility: validation and observed regional differences in the elderly. Journal of Hypertension. 2009;27:535-542.

2. Redheuil A, Wu CO, Kachenoura N, Ohyama Y, Yan RT, Bertoni AG, Hundley GW, Duprez DA, Jacobs DR, Daniels LB, Darwin C, Sibley C, Bluemke DA, Lima JAC. Proximal Aortic Distensibility Is an Independent Predictor of All-Cause Mortality and Incident CV Events. Journal of the American College of Cardiology. 2014;64:2619-2629.

3. Biasiolli L, Hann E, Lukaschuk E, Carapella V, Paiva JM, Aung N, Rayner JJ, Werys K, Fung K, Puchta H, Sanghvi MM, Moon NO, Thomson RJ, Thomas KE, Robson MD, Grau V, Petersen SE, Neubauer S, Piechnik SK. Automated localization and quality control of the aorta in cine CMR can significantly accelerate processing of the UK Biobank population data. PLOS ONE. 2019;14:e0212272.

4. Loh P-R, Tucker G, Bulik-Sullivan BK, Vilhjálmsson BJ, Finucane HK, Salem RM, Chasman DI, Ridker PM, Neale BM, Berger B, Patterson N, Price AL. Efficient Bayesian mixed-model analysis increases association power in large cohorts. Nature Genetics. 2015:47:284-290.

5. Choi SW, O'Reilly PF. PRSice-2: Polygenic Risk Score software for biobank-scale data. Gigascience [Internet]. 2019 [cited 2020 Feb 4];8. Available from: https:// academic.oup.com/gigascience/article/8/7/giz082/5532407

6. Fung K, Ramírez J, Warren HR, Aung N, Lee AM, Tzanis E, Petersen SE, Munroe PB. Genome-wide association study identifies loci for arterial stiffness index in 127,121 UK Biobank participants. Scientific Reports. 2019;9:9143.

7. Sayed-Tabatabaei FA, Van Rijn MJE, Schut AFC, Aulchenko YS, Croes EA, Zillikens MC, Pols HAP, Witteman JCM, Oostra BA, Van Duijn CM. Heritability of the function and structure of the arterial wall: findings of the Erasmus Rucphen Family (ERF) study. Stroke. 2005:36:2351-2356.

8. Mitchell GF, DeStefano AL, Larson MG, Benjamin EJ, Chen M-HH, Vasan RS, Vita $J A$, Levy D. Heritability and a genome-wide linkage scan for arterial stiffness, wave reflection, and mean arterial pressure: The Framingham heart study. Circulation. 2005;112:194-199.

9. Ediger BN, Du A, Liu J, Hunter CS, Walp ER, Schug J, Kaestner KH, Stein R, Stoffers DA, May CL. Islet-1 Is Essential for Pancreatic $\beta$-Cell Function. Diabetes. 2014;63:4206-4217.

10. Stijnen P, Ramos-Molina B, O'Rahilly S, Creemers JWM. PCSK1 Mutations and Human Endocrinopathies: From Obesity to Gastrointestinal Disorders. Endocr Rev. 2016;37:347-371.

\section{THE ROLE OF IMPAIRED MYOCARDIAL MICROVASCULAR FUNCTION DYSGLYCAEMIC PATIENTS WITH HEART FAILURE}

LAE Brown, A Wahab, J Klassen, CED Saunderson, A Das, T Craven, A Chowdhary, N Jex, E Levelt, H Xue, P Kellman, JP Greenwood, S Plein, PP Swoboda.

\subsection{6/heartjnl-2021-BSCMR.10}

Background Dysglycaemic (either diabetic or prediabetic) heart failure patients have worse outcomes than normoglycaemic heart failure patients. It is possible to quantify occult ischaemic heart disease (IHD, either ischaemia on stress perfusion or infarction on late gadolinium enhancement) and myocardial microvascular function (by quantitative perfusion). We aimed to investigate whether excess risk in dysglycaemic patients with heart failure is mediated by occult ischaemic heart disease or myocardial microvascular dysfunction.

Methods We recruited outpatients with a recent diagnosis of heart failure (LVEF $<50 \%$ on echocardiogram). Exclusion criteria included known previous myocardial infarction, revascularisation or angina. Patients were defined as dysglycaemic if they had a previous diagnosis of diabetes or $\mathrm{HbA} 1 \mathrm{c}>42$

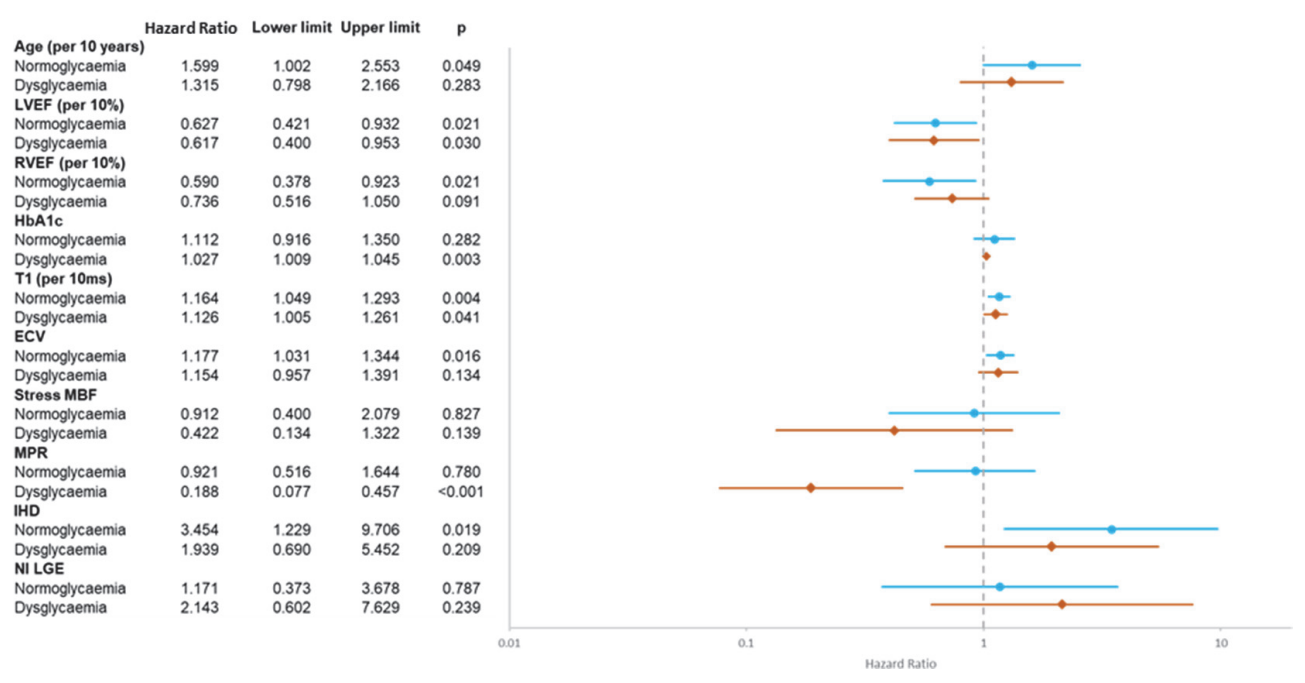

Abstract 10 Figure 1 Univariate Cox regression analysis 